\title{
SUPERHARMONIC TANGENTIAL APPROXIMATION ON LONG ISLANDS
}

\author{
KOHUR GOWRISANKARAN AND ASHOT H. NERSESSIAN
}

\begin{abstract}
We prove uniform and tangential approximation theorems for superharmonic functions in abstract harmonic spaces. Our tangential approximation theorem differs from traditional ones by the absence of the so-called long islands condition. The result is new also in the case of the classical potential theory.
\end{abstract}

\section{Introduction}

Let $\Omega$ be a locally compact, locally connected, connected, second countable topological space. We assume that there is a sheaf of continuous functions satisfying Axioms 1, 2, 3 of Brelot (for more details, see $[4, \mathbf{1 0}, 3]$ ). We also assume that $\Omega$ has a base of determining domains (Axiom D), that 1 is superharmonic, and that there is a positive potential on $\Omega$. Denote by $\Omega^{*}=\Omega \cup\{*\}$ the one-point (Alexandrof) compactification of $\Omega$ by an ideal point *.

For a closed subset $E \subset \Omega$, we denote by $\mathscr{C}^{+}(E)$ the class of strictly positive functions continuous on $E$. Let $\mathscr{H}(E), \mathscr{S}(E)$ represent the classes of functions harmonic and superharmonic, respectively, on $E$ (that is, on an open neighbourhood of $E$ ), while $\mathscr{H}\left(E, E^{o}\right)$ and $\mathscr{S}\left(E, E^{o}\right)$ stand for the classes of functions continuous on $E$ and, respectively, harmonic and superharmonic on the interior $E^{o}$ of the set $E$. As usual, we say that a subset $A \subset \Omega$ is bounded if its closure is a compact subset of $\Omega$. Suppose that $L$ is a closed set. We denote by $\hat{L}$ the union of $L$ and the bounded connected components of $\Omega \backslash L$. The properties of the space $\Omega$ imply its metrisability. Assume that $d(\cdot, \cdot)$ is a metric on $\Omega$, and for a given subset $L \subset \Omega$ and $\delta>0$, denote by $(L)_{\delta}$ the closed $\delta$-neighbourhood of the set $L$ :

$$
(L)_{\delta}=\{x \in \Omega: d(x, L) \leqslant \delta\} .
$$

In [7], the following result is proved.

Theorem A. Suppose that $E$ is a closed subset of the harmonic space $\Omega$. Then the following are equivalent.

(a) For any $u \in \mathscr{S}\left(E, E^{o}\right)$ and $\varepsilon \in \mathscr{C}^{+}(E)$, there exists a function $v \in \mathscr{S}(E)$ such that $u<v<u+\varepsilon$ on $E$.

(b) For any $u \in \mathscr{H}\left(E, E^{o}\right)$ and $\varepsilon \in \mathscr{C}^{+}(E)$, there exists a function $v \in \mathscr{H}(E)$ such that $u<v<u+\varepsilon$ on $E$.

(c) (i) $\Omega \backslash E$ and $\Omega \backslash E^{o}$ are thin at the same points of $E$.

(ii) For each compact subset $K$ of $\Omega$, there exists another compact subset $L$ of $\Omega$ such that all components of $E^{o}$ having non-empty intersection with $K$ are contained in $L$.

Received 14 January 1998; revised 13 September 1998.

1991 Mathematics Subject Classification 31D05.

Bull. London Math. Soc. 32 (2000) 47-53 
In the part referring to harmonic functions, this result was first proved for the classical case, for which it was also shown that approximation from above is equivalent to approximation in modulus (see [5, Theorem 4]). We refer to [6] for an account of classical harmonic and superharmonic approximation.

Condition (c)(ii) is called the long islands condition. We show that tangential approximation of a different, quite natural type (see statement (a) in Theorem 1 below) in the case of superharmonic functions does not imply tangential approximation for harmonic functions as in Theorem A.

As usual, we say that the sequence $\left(\Omega_{k}\right)_{k=1}^{\infty}$ of compact subsets of $\Omega$ constitutes a compact exhaustion of $\Omega$ if (a) $\Omega_{k} \subset\left(\Omega_{k+1}\right)^{o}, k=1,2, \ldots$, and (b) $\bigcup_{k=1}^{\infty} \Omega_{k}=\Omega$.

In what follows, we consider unbounded subsets $E \subset \Omega$ satisfying the following condition.

(A) Suppose that $\left(\Omega_{k}\right)_{k=1}^{\infty}$ is an exhaustion of $\Omega$ by compact subsets such that $\widehat{\Omega_{k}}=\Omega_{k}$. Then for any $k \geqslant 1$, every connected component of $\Omega \backslash \Omega_{k}$ has non-void intersection with $\Omega \backslash E$.

It is immediate that condition (A) is independent of the choice of the exhaustion by compact subsets with the property $\widehat{\Omega_{k}}=\Omega_{k}$.

We now state the main result of this paper.

THEOREM 1. Suppose that $E$ is a closed subset of the harmonic space $\Omega$ satisfying condition $(A)$. Then the following are equivalent.

(a) For any $u \in \mathscr{S}\left(E, E^{o}\right)$ and $\varepsilon \in \mathscr{C}^{+}(E)$, there exists a function $v \in \mathscr{S}(E)$ such that $|u-v|<\varepsilon$ on $E$.

(b) For any $u \in \mathscr{S}\left(E, E^{o}\right)$ and positive number $\delta$, there exists a function $v \in \mathscr{S}(E)$ such that $u<v<u+\delta$ on $E$.

(c) For any $u \in \mathscr{H}\left(E, E^{o}\right)$ and positive number $\delta$, there exists a function $v \in \mathscr{H}(E)$ such that $u<v<u+\delta$ on $E$.

(d) $\Omega \backslash E$ and $\Omega \backslash E^{o}$ are thin at the same points on $E$. disc.

We give an example for the classical case of superharmonic functions in the unit

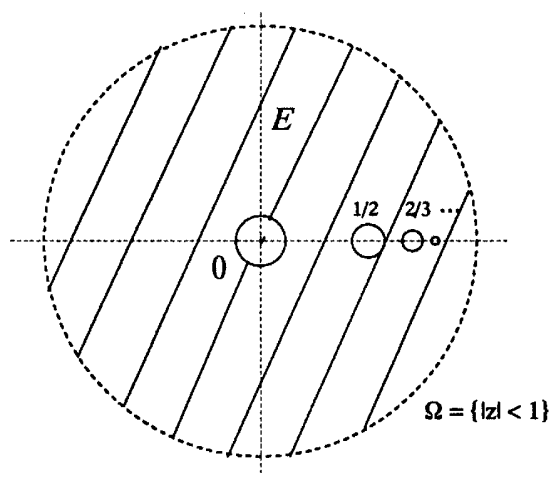

FIG. 1 
EXAMPLE 1. Taking $\Omega=\{|z|<1\}$ and

$$
E=\Omega \backslash \bigcup_{n=1}^{\infty}\left\{\left|z-\left(1-\frac{1}{n}\right)\right|<\frac{1}{2(n+1)^{2}}\right\},
$$

we obtain an example of a long island (a closed set with a unique 'unbounded' connected interior) which is a tangential approximation set with arbitrary speed (see Fig. 1). On the other hand, for the same space $\Omega=\{|z|<1\}$ (or for the space $\Omega=\{0<|z|<1\})$ and $E=\Omega \backslash\{|z|<1 / 8\}$, we are in a situation where the complement of $E$ in $\Omega$ does not satisfy condition (A), and for this case our Theorem 1 does not provide any information. The set $E$ filling out the vicinity of the 'ideal' boundary of $\Omega$ (or some component of it) does not allow for a superharmonic function to decrease by modulus to zero with arbitrary speed. That a positive superharmonic function cannot converge arbitrarily rapidly to zero is shown in [7]. Our conjecture is that the necessary oscillation will force the superharmonic function to produce minima which are not permitted. We have been unable to give a complete answer for this case.

The following statement deals with the case of tangential approximation by globally superharmonic functions.

TheOREM 2. Let $\Omega$ and $E$ be as in Theorem 1. Then the following are equivalent.

(a) For any $u \in \mathscr{S}\left(E, E^{o}\right)$ and $\varepsilon \in \mathscr{C}^{+}(E)$, there exists a function $v \in \mathscr{S}(\Omega)$ such that $|u-v|<\varepsilon$ on $E$.

(b) For any $u \in \mathscr{S}\left(E, E^{o}\right)$ and positive number $\delta$, there exists a function $v \in \mathscr{S}(\Omega)$ such that $u<v<u+\delta$ on $E$.

(c) (i) $\Omega \backslash \hat{E}$ and $\Omega \backslash E^{o}$ are thin at the same points on $E$.

(ii) $\Omega^{*} \backslash \hat{E}$ is locally connected at *.

REMARK 1. The case of compact subsets $E$ in both Theorems 1 and 2 obviously reduces to uniform approximation. Therefore, in the proofs below it is assumed that $E$ is a closed non-compact (that is, non-bounded) subset of $\Omega$. Notice that the proof of necessity of (c)(ii) in Theorem A is essentially based on the strictness of the inequality in (a) (and/or (b)) in Theorem A. This agrees with the situation in Theorems 1 and 2, asserting that the less demanding character of the estimate $v-\varepsilon<u<v+\varepsilon$ makes, in fact, condition (c)(ii) redundant.

REMARK 2. That there is no harmonic analogue to the equivalence between properties (a) and (c) in the above Theorems 1 and 2 (under whatever further restrictions on the space $\Omega$ ) follows at once from the Theorem in [1] (see also the final remark there). We note also that in every statement on tangential approximation with arbitrary speed known to the authors thus far, be it in the complex analytic or in classical or abstract harmonic cases, the long islands condition has been essential.

REMARK 3. The construction applying Lemma 1 below obviously produces analogies to the widely known fusion lemma type statements. Fusion statements in abstract harmonic spaces, along with their application to approximation problems, will be discussed in a later paper. 


\section{Proof of Theorem 1}

The implication $(a) \Rightarrow(b)$ is obvious. The implication $(b) \Rightarrow(c)$ is achieved in a routine way (see, for instance, [7]), and the implication $(c) \Rightarrow(d)$ is part of the theorem on uniform approximation, proved exactly as in [7]. (One has to take $h=1$ in the reasoning on page 1010 of [7].) It remains to prove the implication (d) $\Rightarrow(\mathrm{a})$.

We shall make use of the following.

Proposition 1. There exists an exhaustion $\left(\Omega_{k}\right)$ of $\Omega$ by compact subsets such that for every $k \geqslant 0$, the set $\left(\Omega_{2 k+1}\right)^{o} \backslash \Omega_{2 k}$ consists of only a finite number of connected components all of which have non-empty intersection with $\Omega \backslash E$.

Proof. Notice first that for any compact exhaustion $\left(L_{k}\right)_{k \geqslant 0}$ of $\Omega$, the sequence $\left(\widehat{L_{k}}\right)_{k \geqslant 0}$ also represents an exhaustion. Hence we can assume in advance that the given exhaustion $\left(L_{k}\right)_{k \geqslant 0}$ is such that $\widehat{L_{k}}=L_{k}$, for $k \geqslant 0$. From $L_{k} \subset\left(L_{k+1}\right)^{o}$, it follows that there exist $\delta_{k}>0$ such that $\left(L_{k}\right)_{\delta_{k}} \subset\left(L_{k+1}\right)^{o}$, and also $\left(\widehat{\left.L_{k}\right)_{\delta_{k}}} \subset\left(L_{k+1}\right)^{o}\right.$ because $\widehat{L_{k+1}}=L_{k+1}$. We see that $\left(\left(\widehat{\left.L_{k}\right)_{\delta_{k}}}\right)_{k \geqslant 0}\right.$ is an exhaustion of $\Omega$. Assume that for some $k$, the interior of $\left(\widehat{\left.L_{k}\right)_{\delta_{k}}}\right.$ has infinitely many connected components. Since every such component will contain points of $L_{k}$, we can choose a sequence $\left(x_{n}\right)_{n \geqslant 1} \subset L_{k}$ of points belonging to distinct components of the interior of $\left(\bar{L}_{k}\right)_{\delta_{k}}$. By the sequential compactness of the space, we can assume that $x_{n} \rightarrow x_{0}$ for some point $x_{0} \in L_{k}$. On the other hand, because of the local connectedness of the space, there exists a (possibly smaller) connected neighbourhood of $x_{0}$ in its $\delta_{k}$-neighbourhood. Hence all $x_{n}$ from some number on belong to the same connected component of $\left(\widehat{\left.L_{k}\right)_{\delta_{k}}}\right.$, and this is in contradiction with the $x_{n}$ belonging to distinct components.

We now show that for any exhaustion $\left(L_{k}\right)_{k \geqslant 0}$ with the condition $\widehat{L_{k}}=L_{k}$, $k \geqslant 0$, the sets $\Omega \backslash L_{k}$ can have no more than a finite number of connected components. Assume that this fails for some $L_{k}$. There exists a $\delta>0$ such that $\left(L_{k}\right)_{\delta}$ is precompact. In each connected component of $\Omega \backslash L_{k}$, we take a point $x_{n}$ such that $\delta / 2 \leqslant d\left(x_{n}, L_{k}\right) \leqslant \delta$. The existence of such points follows from the connectedness of the space. By the sequential compactness property, we may assume that $x_{n} \rightarrow x_{0}$ for some $x_{0} \in \Omega$. Now, on the one hand we have $\delta / 2 \leqslant d\left(x_{0}, L_{k}\right)$, and, since the points $x_{n}$ are separated from each other by the set $L_{k}$ because of the connectedness of $\Omega$, we have also $x_{0} \in L_{k}$, which gives a contradiction.

Now, if $L_{k}$ is an exhaustion for which both $\left(L_{k}\right)^{o}$ and $\Omega \backslash L_{k}$ consist of no more than a finite number of connected components, then this same property will hold also for the sets $\left(L_{n}\right)^{o} \backslash L_{k}$ for any $n$ and $k$ with $n \geqslant k+1$.

In view of condition (A), taking as $\left(\Omega_{k}\right)_{k}$ either the exhaustion $\left(L_{k}\right)_{k}$ itself or one of its appropriate subsequences, we shall arrive at an exhaustion having the required properties.

We assume that $\left(\Omega_{k}\right)$ is an exhaustion as in Proposition 1 , and in addition let $\Omega_{-1}=\Omega_{-2}=\Omega_{-3}=\varnothing$. Denote $A[k, m]=\Omega_{m} \backslash \Omega_{k}^{o}, 0 \leqslant k<m, m \geqslant 1$.

The following result provides the main tool for the proof of Theorem 1 .

Lemma 1. For any $k \geqslant 0$, there exists a function $\chi_{k} \in \mathscr{S}(E \cap A[2 k-3,2 k+2])$ such that

$$
\chi_{k}= \begin{cases}0 & \text { on } E \cap A[2 k-1,2 k], \\ 1 & \text { on } E \cap(A[2 k-3,2 k-2] \cup A[2 k+1,2 k+2]) .\end{cases}
$$


Proof. For a given $k \geqslant 0$, take a regular neighbourhood $L$ of

$$
E \cap(A[2 k-2,2 k-1] \cup A[2 k, 2 k+1])
$$

such that every component of the set $(A[2 k-2,2 k-1] \cup A[2 k, 2 k+1])^{o}$ has nonempty intersection with the set $\Omega \backslash \bar{L}$. Let $U_{1}, U_{2}$ be disjoint open neighbourhoods of, respectively, $M=A[2 k-3,2 k-2] \cup A[2 k+1,2 k+2]$ and $A[2 k-1,2 k]$. We can choose $U_{1}, U_{2}$ in such a way (shrinking them if necessary) that every component of $(A[2 k-2,2 k-1] \cup A[2 k, 2 k+1])^{o} \backslash\left(U_{1} \cup U_{2}\right)$ (the number of which is assumed to be finite due to the choice of the exhaustion) has non-empty intersection with the set $\Omega \backslash \bar{L}$.

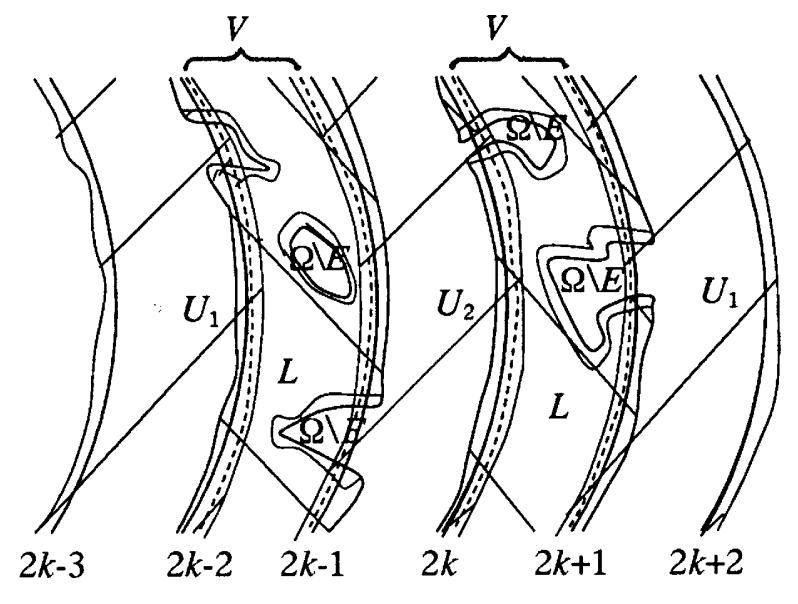

FIG. 2

Further, let $V$ be a regular open neighbourhood of $A[2 k-3,2 k+2] \backslash\left(U_{1} \cup U_{2}\right)$ not intersecting $M \cup A[2 k-1,2 k]$. Since the points on $\partial L$ are regular for $\tilde{V}=V \cap L$, the harmonic measure of $\partial L$ is strictly positive on $\partial\left(U_{1} \cup U_{2}\right) \cap \tilde{V}$ in every component of $\tilde{V}$. Let $s$ be the solution of the generalised Dirichlet problem, for boundary values equal to 2 on $\partial \tilde{V} \cap \partial V$, and so small on $\partial L \cap \partial \tilde{V}$ that $s$ is negative on $\partial\left(U_{1} \cup U_{2}\right) \cap \tilde{V}$. Now define

$$
\chi_{k}= \begin{cases}s & \text { on } \tilde{V} \backslash\left(U_{1} \cup U_{2}\right), \\ 1 & \text { on } U_{1} \backslash V, \\ 0 & \text { on } U_{2} \backslash V, \\ \min (1, s) & \text { on } U_{1} \cap \tilde{V}, \\ \min (0, s) & \text { on } U_{2} \cap \tilde{V}\end{cases}
$$

Obviously, the functions $\chi_{k}$ have the properties listed in the lemma.

Returning to the proof of Theorem 1 , suppose that $\left(C_{k}\right)$ is an increasing sequence such that $C_{k} \geqslant \max _{A[2 k-3,2 k+2]}\left|\chi_{k}\right|$. Further, assume that $\left(\varepsilon_{k}\right)$ is a decreasing sequence of positive numbers such that

$$
\varepsilon_{k}<\frac{1}{4 C_{k+2}} \min _{A[2 k-1,2 k+4]}(\varepsilon), \quad k \geqslant 0 .
$$

Recall that the compact set $L$ is called stable if $\Omega \backslash L$ is not thin at any point on $\partial L$. According to [7, Lemma 1], for any $k \geqslant 0$ there exists a stable compact set $\Omega_{k}^{\prime}$ 
such that $\Omega_{k} \subset\left(\Omega_{k}^{\prime}\right)^{o}$ and $\Omega_{k}^{\prime} \subset \Omega_{k+1}^{o}$. Making use of condition (d) and the theorem on uniform approximation on compact sets [2, Theorem 4.7], we conclude that for any $k \geqslant 0$ there exists a function $h_{k} \in \mathscr{S}\left(E \cap \Omega_{2 k+2}^{\prime}\right)$ such that

$$
\left|u-h_{k}\right|<\varepsilon_{k} \quad \text { on } E \cap \Omega_{2 k+2}^{\prime} .
$$

Put $\tilde{h}_{k}=h_{k}+\left(\varepsilon_{k-1}+\varepsilon_{k}+\varepsilon_{k+1}\right) \chi_{k}$ on a neighbourhood $W_{k}$ of $E \cap A[2 k-3,2 k+2]$, where $h_{k}$ and $\chi_{k}$ are both superharmonic. This definition implies that for all $k \geqslant 0$,

$$
\tilde{h}_{k-1} \quad \text { and } \quad \tilde{h}_{k+1}>\tilde{h}_{k} \quad \text { on } E \cap A[2 k-1,2 k]
$$

(we assume $h_{-1}=\infty$ ). Indeed, for $k \geqslant 1$ we have, on $E \cap A[2 k-1,2 k]$,

$$
\begin{aligned}
\tilde{h}_{k-1} & =h_{k-1}+\varepsilon_{k-2}+\varepsilon_{k-1}+\varepsilon_{k} \\
& >u+\varepsilon_{k-2}+\varepsilon_{k} \\
& >h_{k}=\tilde{h}_{k} .
\end{aligned}
$$

And for $k \geqslant 0$ we have, on $E \cap A[2 k-1,2 k]$,

$$
\begin{aligned}
\tilde{h}_{k+1} & =h_{k+1}+\varepsilon_{k}+\varepsilon_{k+1}+\varepsilon_{k+2} \\
& >u+\varepsilon_{k}+\varepsilon_{k+2} \\
& >h_{k}=\tilde{h}_{k} .
\end{aligned}
$$

Since the functions $\tilde{h}_{k}$ are continuous on $W_{k}$, we may assume that the inequalities (2.4) are indeed satisfied on $W_{k-1} \cap W_{k} \cap W_{k+1} \cap A[2 k-1,2 k]$, adjusting the sets $W_{k}$, if necessary. Define $\tilde{h}_{k}=\infty$ off $W_{k} \cap A[2 k-3,2 k+2]$, and put $h=\inf _{k \geqslant 0} \tilde{h}_{k}$ on $W=\bigcup_{k \geqslant 0} W_{k}$. Since on any $W \cap A[k, k+1]$ only at most three functions $\tilde{h}_{k}$ are finite, and they are also superharmonic there, the function $h$ is superharmonic on $W$. For any $k \geqslant 0$, due to (2.4) and since for $j \leqslant k-2$ and $j \geqslant k+1, \tilde{h}_{j}=\infty$ on $A[2 k-2,2 k-1]$, we have $h=\min \left(\tilde{h}_{k-1}, \tilde{h}_{k}\right)$ on $W \cap A[2 k-2,2 k]$. By the same reasoning, $h=\min \left(\tilde{h}_{k}, \tilde{h}_{k+1}\right)$ on $W \cap A[2 k-1,2 k+1]$. Since both of these functions are continuous and superharmonic in the interiors of these sets, and coincide on $W \cap A[2 k-1,2 k]$ (they are both equal to $\tilde{h}_{k}$ ), we conclude that $h \in \mathscr{S}(W) \subset \mathscr{S}(E)$.

At points of $E \cap A[2 k-1,2 k]$, due to (2.4) we have $h=\tilde{h}_{k}=h_{k}$, hence $|u-h|<\varepsilon_{k}$.

On $E \cap A[2 k, 2 k+1]$ we have, in view of (2.2) and (2.3),

$$
\begin{aligned}
|u-h| & \leqslant \max \left\{\left|u-\tilde{h}_{k}\right|,\left|u-\tilde{h}_{k+1}\right|\right\} \\
& <\max \left\{\varepsilon_{k}+\left(\varepsilon_{k-1}+\varepsilon_{k}+\varepsilon_{k+1}\right) C_{k}, \varepsilon_{k+1}+\left(\varepsilon_{k}+\varepsilon_{k+1}+\varepsilon_{k+2}\right) C_{k+1}\right\} \\
& \leqslant \varepsilon_{k}+\left(\varepsilon_{k-1}+\varepsilon_{k}+\varepsilon_{k+1}\right) C_{k+1} \\
& <\frac{1}{4 C_{k+2}} \min _{A[2 k, 2 k+1]}(\varepsilon)+\frac{3}{4 C_{k+1}} C_{k+1} \min _{A[2 k, 2 k+1]}(\varepsilon) \\
& <\min _{A[2 k, 2 k+1]}(\varepsilon) .
\end{aligned}
$$

Theorem 1 is proved.

Remark 4. For an arbitrary $\varepsilon \in \mathscr{C}^{+}(E)$, let $W_{k}$ be a neighbourhood of $E \cap A[2 k-3,2 k+2]$, where $\chi_{k}$ is superharmonic. Taking $\tilde{h}_{k}=\left(\varepsilon_{k-1}+\varepsilon_{k}+\varepsilon_{k+1}\right) \chi_{k}$ on $W_{k} \cap A[2 k-3,2 k+2]$ and equal to $\infty$ off this same set for $\varepsilon_{k}$ chosen according to (2.2), and defining $h=\inf _{k \geqslant 0} \widetilde{h}_{k}$, we obtain a function $h \in \mathscr{S}(E)$ such that $|h|<\varepsilon$ on $E$. 


\section{Proof of Theorem 2}

The implication $(\mathrm{a}) \Rightarrow(\mathrm{b})$ is obvious. $(\mathrm{b}) \Rightarrow(\mathrm{c})(\mathrm{i})$ is proved as in Theorem 3 of [9] (or as in [7], taking the function $h=1$ on page 1010). (b) $\Rightarrow$ (c)(ii) is proved as in Theorem 4 of [9], taking into account that the proof in fact uses only uniform approximation. It remains to prove the implication $(\mathrm{c}) \Rightarrow(\mathrm{a})$.

Thanks to condition (c)(i), any function $u \in \mathscr{S}\left(E, E^{o}\right)$ continues to a function $\tilde{u} \in \mathscr{S}\left(\hat{E},(\hat{E})^{o}\right)$ (see [8]). Since from (c)(i) it also follows that $\Omega \backslash \hat{E}$ and $\Omega \backslash(\hat{E})^{o}$ are thin at the same points on $E$, and hence on $\hat{E}$, by Theorem 1 there exists a function $v \in \mathscr{S}(\hat{E})$ satisfying $|\tilde{u}-v|<\varepsilon$ on $\hat{E}$. By Theorem 1 in [9], thanks to (c)(ii) this function can be extended continuously from a neighbourhood of $\hat{E}$ to a function in $\mathscr{S}(\Omega)$.

Theorem 2 is proved.

\section{References}

1. D. H. Armitage, T. Bagby and P. M. Gauthier, 'Note on the decay of solutions of elliptic equations', Bull. London Math. Soc. 17 (1985) 554-556.

2. J. Bliedtner and W. Hansen, 'Symplicial cones in potential theory II (Approximation theorems)', Invent. Math. 46 (1978) 255-275.

3. J. Bliedtner and W. Hansen, Potential theory, an analytic and probabilistic approach to balayage (Springer, Berlin, 1986).

4. M. Brelot, Potential theory (Tata Institute, Bombay, 1960).

5. S. J. Gardiner, 'Tangential harmonic approximation on relatively closed sets', Illinois J. Math. 39 (1995) 143-157.

6. S. J. Gardiner, Harmonic approximation, London Math. Soc. Lecture Note Ser. 221 (Cambridge University Press, 1995).

7. S. J. Gardiner, M. Goldstein and K. GowriSankaran, 'Tangential harmonic approximation', Indiana Univ. Math. J. 43 (1994) 1003-1012.

8. S. Gardiner, M. Goldstein and K. GowriSankaran, 'Global approximation in harmonic spaces', Proc. Amer. Math. Soc. 122 (1994) 213-221.

9. K. GowriSankaran and A. H. Nersessian, 'Superharmonic extension and tangential approximation in harmonic spaces', in preparation.

10. R. M. Hervé, 'Recherches axiomatiques sur la théorie des fonctions surharmoniques et du potentiel', Ann. Inst. Fourier (Grenoble) 12 (1962) 415-571.

Burnside Hall

Department of Mathematics

and Statistics

McGill University

Montreal H3A 2K6

Canada 